\title{
Simple synthesis of porous melamine-formaldehyde resins by low temperature solvothermal method and its $\mathrm{CO}_{2}$ adsorption properties
}

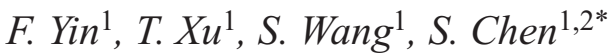 \\ ${ }^{1}$ PCFM Lab, School of Chemistry, Sun Yat-Sen University, 510275 Guangzhou, PR China \\ ${ }^{2}$ Materials Science Institute, Sun Yat-Sen University, 510275 Guangzhou, PR China
}

Received 23 March 2017; accepted in revised form 11 June 2017

\begin{abstract}
A simple and environmentally-friendly approach for the preparation of porous melamine-formaldehyde resins (PMFRs) was developed by using low-boiling-point solvents, such as water, as pore-forming agent. With using dimethyl sulfoxide (DMSO) and low-boiling solvents cosolvent method, PMFRs with a high specific surface area and well-defined pore structure can be synthesized at a low reaction temperature of $140^{\circ} \mathrm{C}$ for a short reaction duration in 20 hours, which can replace the conventional methods that use dimethyl sulfoxide (DMSO) as reaction medium and require 3 days at $170^{\circ} \mathrm{C}$ to achieve similar surface area. When loaded with polyethylenimine (PEI), the PMFR-PEI- $30 \%$ showed good $\mathrm{CO}_{2}$ adsorption performance with a capacity of up to $2.89 \mathrm{mmol} / \mathrm{g}$ at $30^{\circ} \mathrm{C}$. These results bring new perspectives for the development of lowcost and environmentally-friendly synthetic methods for porous materials, which can boost their widespread applications.
\end{abstract}

Keywords: polymer synthesis, molecular engineering, porous melamine-formaldehyde resins, low-boiling-point solvents, low temperature, $\mathrm{CO}_{2}$ adsorption

\section{Introduction}

The ever-increasing carbon dioxide $\left(\mathrm{CO}_{2}\right)$ emission from burning fossil fuels has been regarded as the major cause of climate change and global warming [1-4]. To mitigate the effect of global warming, many porous materials, such as metal organic frameworks (MOFs) [5-9], porous co-ordination polymers [10, 11], porous carbons [12-16], porous organic polymers (POPs) and covalent organic frameworks (COFs) [17-20], have been investigated for $\mathrm{CO}_{2}$ storage.

In recent years, many amine functionalized or nitrogen-rich porous materials were found to show good $\mathrm{CO}_{2}$ adsorption properties because of the existence of strong interactions between the nitrogen functionality and $\mathrm{CO}_{2}$ molecules [21-27]. Among them, the porous melamine-formaldehyde resins (PMFRs) have gained great attention because they can be produced by low-cost highly abundant melamine that has suitable alkalescence $(p K a=5.5)$ versatile reactivity [28-30]. In addition, it is a mass produced product in the chemical industry and can be used as crosslinker in organic coating and plastic [31-33]. In general, the PMFRs can be synthesized by using the microemulsion approach or the template method, such as $\mathrm{SiO}_{2}$ particles [34]. However, PMFRs produced by these approaches normally have a low BrunauerEmmett-Teller (BET) specific surface area, which were listed in Table $1[28,31]$. When used for $\mathrm{CO}_{2}$ adsorption, their capacity is typically not exceeding $1.6 \mathrm{mmol} / \mathrm{g}\left(273 \mathrm{~K}, \mathrm{CO}_{2}\right.$ partial pressure 0.03$)$. To tackle with this problem, Tan et al. [35] developed a simple one-step synthesis method for PMFRs by using the polycondensation reaction with the dimethyl sulfoxide (DMSO) as the solvent. The as-obtained 
Table 1. The comparative description of the synthesis and product properties of types PMFR

\begin{tabular}{|l|c|c|c|c|}
\hline $\begin{array}{c}\text { Pore-forming } \\
\text { agent }\end{array}$ & $\begin{array}{c}\text { BET specific } \\
\text { surface area } \\
{\left[\mathbf{m}^{\mathbf{2}} \mathbf{g}\right]}\end{array}$ & $\begin{array}{c}\text { Pore } \\
\text { volume } \\
{\left[\mathbf{c m}^{\mathbf{3}} / \mathbf{g}\right]}\end{array}$ & $\begin{array}{c}\text { Average } \\
\text { pore width } \\
{[\mathbf{n m}]}\end{array}$ & Ref. \\
\hline $\mathrm{F} 127$ & 258 & 0.53 & 7.8 & 28 \\
\hline $\mathrm{SiO}_{2}$ & 234 & 1.05 & 17.4 & 31 \\
\hline Solvent & 1099 & 2.09 & 10.3 & 35 \\
\hline
\end{tabular}

PMFRs exhibit greatly increased specific surface area up to $1099 \mathrm{~m}^{2} / \mathrm{g}$ and have demonstrated a large $\mathrm{CO}_{2}$ adsorption capacity of $15.3 \mathrm{wt} \%(273 \mathrm{~K}, 1 \mathrm{~atm})$. However, the synthesis requires high temperature and long time, e.g., $170^{\circ} \mathrm{C}$ and 72 hours, leading to high synthesis costs and low productivity. Under such high temperatures, the DMSO tends to decompose and release toxic products, such as formaldehyde and some sulfides. This is not only bad for the environment but also makes the post-treatment difficult because it needs a large amount of organic solvent (DMSO, acetone, THF and $\mathrm{CH}_{2} \mathrm{CH}_{2}$ ) to purification. In this study, a simple low-temperature and environmentally-friendly synthesis method for porous materials was developed by using low-boiling-point solvents, such as water, dioxane, methanol and ethanol, as pore-forming agent. With this method, PMFRs with a high specific surface area, well-defined pore structure and good $\mathrm{CO}_{2}$ adsorption performance can be synthesized at a low temperature. When loaded with polyethylenimine (PEI), the PMFR-PEI-30\% show good $\mathrm{CO}_{2}$ adsorption and regeneration performance. This research brings a new perspectives to develop a new synthesis method and provides useful information for understanding the synthesis process of PMFRs.

\section{Experimental}

\subsection{Materials}

Melamine (AR), polydivinyl benzene and poly (ethylenimine) (PEI, $M_{\mathrm{w}}=600$ ) were purchased from Aladdin Chemistry Co. Ltd, China. Paraformaldehyde, dimethyl sulfoxide (DMSO, AR) and ethyl alcohol (AR) were from Tianjin-Fuchen Chemical Reagent Factory, China. Acetone (AR), N,N-dimethylformamide (AR), ethylacetate (AR), dioxane (AR) and tetrahydrofuran(THF, AR) were purchased from Guangzhou Chemical Reagent Factory, China. All reagents were used without further purification.

\subsection{The synthesis of porous melamine- formaldehyde resins (PMFRs)}

In a typical preparation process, melamine $(2.25 \mathrm{~g}$, $17.8 \mathrm{mmol})$ and paraformaldehyde $(1.34 \mathrm{~g}$, $44.6 \mathrm{mmol}$ ) were dissolved in $20 \mathrm{~mL}$ DMSO or the same volume of mixed solvent of DMSO and $\mathrm{H}_{2} \mathrm{O} /$ or dioxane, the volume ratio of DMSO to $\mathrm{H}_{2} \mathrm{O}$ /or dioxane was 15:5. The mixture was stirred until it became transparent, and was then carefully transferred into a Teflon container and kept at 140 or $170^{\circ} \mathrm{C}$ for 20 hours to complete the polycondensation reaction of melamine and paraformaldehyde. The polycondensation product, a white solid was ground, sieved, and then successively washed with ethyl alcohol. The obtained granules were dried under vacuum at $80^{\circ} \mathrm{C}$, and noted as PMFRs (the formation mechanism was shown in Figure 1). The resulting samples were denoted as PMFR-S-T (for example, PMFR-DMSO $+\mathrm{H}_{2} \mathrm{O}-140{ }^{\circ} \mathrm{C}$ ), where $\mathrm{S}$ represents the synthesis medium (DMSO, $\mathrm{H}_{2} \mathrm{O}$ or dioxane), $T$ represents the reaction temperature.<smiles>CC#CCNc1nc(NC)nc(NCNc2nc(NC)nc(NCO)n2)n1</smiles>

Figure 1. The formation mechanism of PMFRs 
In order to investigate the effect of water content in the mix solvent, the monomer concentration and the molar ratio of paraformaldehyde to melamine on the porosities of the obtained PMFRs, volume ratio of water to DMSO would be adjusted from 0:20 to $10: 10$, the total monomer concentration of paraformaldehyde and melamine were adjusted from 3.15 to $5.0 \mathrm{mmol} / \mathrm{mL}$, and the molar ratio of paraformaldehyde to melamine was from 2.0 to 3.5 .

\subsection{Preparation of PEI impregnated adsorbent (PMFR-PEI)}

PEI was loaded onto PMFRs by using a wet impregnation method. 0.1-0.5 g PEI was dissolved in $20 \mathrm{~mL}$ ethanol, and then 0.9-0.5 g PMFR was added into the solution. The mixture was continuously stirred at room temperature for $3 \mathrm{~h}$. After being dried at $60^{\circ} \mathrm{C}$ for $12 \mathrm{~h}$, the adsorbent was obtained and is denoted as PMFR-PEI-X\%, where $\mathrm{X} \%$ is used to identify the weight percentage of $\mathrm{PEI}$ in the adsorbent and is normally within the range of $10 \sim 50 \%$, as showing Table 2.

\subsection{Characterization}

Nitrogen adsorption-desorption isotherms was characterized at $77.35 \mathrm{~K}$ on an automatic gas adsorption instrument (ASAP2020, Micromeritics Corp., USA) at the range of relative pressure from $10^{-6}$ to 1 . Before each test, the sample was dried under vacuum at $100^{\circ} \mathrm{C}$ overnight. $V_{\text {total }}$ was calculated based on the nitrogen amount adsorbed at $P / P_{0}=0.95$. Specific surface area and pore parameters were calculated using the Brunauer-Emmett-Teller (BET) method and density functional theory (DFT) method, respectively. Scanning electron microscope (SEM, S4800, Hitachi, Japan) was used to observe the morphology and microstructure of the samples, and transmission electron microscope (TEM, JEM-2010HR, JEOL, Japan) was applied to observe the porous structure.

Table 2. Dosage of PEI and porous amino resin for amine impregnation

\begin{tabular}{|c|c|c|c|}
\hline $\begin{array}{c}\text { Total mass } \\
\mathbf{[ g}]\end{array}$ & $\begin{array}{c}\text { PEI } \\
\mathbf{[ g}]\end{array}$ & $\begin{array}{c}\text { Porous amino resin } \\
\mathbf{[ g ]}\end{array}$ & $\begin{array}{c}\text { Amine loading } \\
{[\mathbf{\%}]}\end{array}$ \\
\hline 1.00 & 0.10 & 0.90 & 10 \\
\hline 1.00 & 0.20 & 0.80 & 20 \\
\hline 1.00 & 0.30 & 0.70 & 30 \\
\hline 1.00 & 0.40 & 0.60 & 40 \\
\hline 1.00 & 0.50 & 0.50 & 50 \\
\hline
\end{tabular}

\subsection{The $\mathrm{CO}_{2}$ adsorption measurement}

The $\mathrm{CO}_{2}$ adsorption measurement was performed in a fixed bed flow system. After the sample was dried under vacuum at $80^{\circ} \mathrm{C}$ for $12 \mathrm{~h}, 0.5 \mathrm{~g}$ of adsorbent was loaded into a glass tube $(\Phi=1.3 \mathrm{~cm})$. To remove the air and residue water, dry nitrogen was introduced into the column at a flow rate of $30 \mathrm{~mL} / \mathrm{min}$ for 20 minutes while the column was kept at $90^{\circ} \mathrm{C}$. Then a mixed gas, with $10 \% \mathrm{CO}_{2}$ and $90 \% \mathrm{~N}_{2}$, was introduced to the tube at a flow rate of $30 \mathrm{~mL} / \mathrm{min}$ for the adsorption test. the $\mathrm{CO}_{2}$ concentrations at the inlet and outlet were determined by an Agilent 6820 gas chromatography that equipped with a thermal conductivity detector. After $\mathrm{CO}_{2}$ adsorption, the adsorbent was regenerated by purging with $\mathrm{N}_{2}$ with a flow rate of $30 \mathrm{~mL} / \mathrm{min}$ at $90^{\circ} \mathrm{C}$.

The adsorption amount was calculated as shown by Equation (1):

$Q=\int_{0}^{\mathrm{t}} \frac{\left(C_{\mathrm{in}}-C_{\text {eff }}\right) \cdot V}{22.4 W} \mathrm{~d} t$

where $Q$ is the adsorption capacity of the adsorbent $[\mathrm{mmol} \mathrm{CO} / \mathrm{g}], t$ is the adsorption time [min], and $C_{\text {in }}$ and $C_{\text {eff }}$ are the influent and effluent flow rate of $\mathrm{CO}_{2}[\mathrm{~mL} / \mathrm{min}]$, respectively; $V$ is the total flow rate, [30 $\mathrm{mL} / \mathrm{min}] ; W$ and 22.4 are the weight of the adsorbent $[\mathrm{g}]$ and molar volume of gas $[\mathrm{mmol} / \mathrm{mL}]$, respectively.

\section{Results and discussion}

\subsection{Preparation and pore tailoring of PMFRs}

PMFRs were first synthesized by the polycondensation of melamine and paraformaldehyde in DMSO system at $140^{\circ} \mathrm{C}\left(\mathrm{PMFR}-\mathrm{DMSO}-140^{\circ} \mathrm{C}\right.$ ) and $170^{\circ} \mathrm{C}$ (PMFR-DMSO- $170^{\circ} \mathrm{C}$ ), respectively. Their specific surface area and pore structures were compared in Figure 2 and Table 3 . The specific surface area of PMFR-DMSO- $170^{\circ} \mathrm{C}$ is almost three times as large as that of PMFR-DMSO- $140^{\circ} \mathrm{C}$. PMFR-DMSO$170^{\circ} \mathrm{C}$ also show more disordered pore-size distribution. This clearly indicates that the higher temperature can promote the formation of pores. It has been reported that at a high temperature DMSO can decompose into low-boiling-point small molecules, such as formaldehyde and dimethylsulfide. These small molecules could act as pore-forming agent and promote the formation of porous structure [36, 37]. 

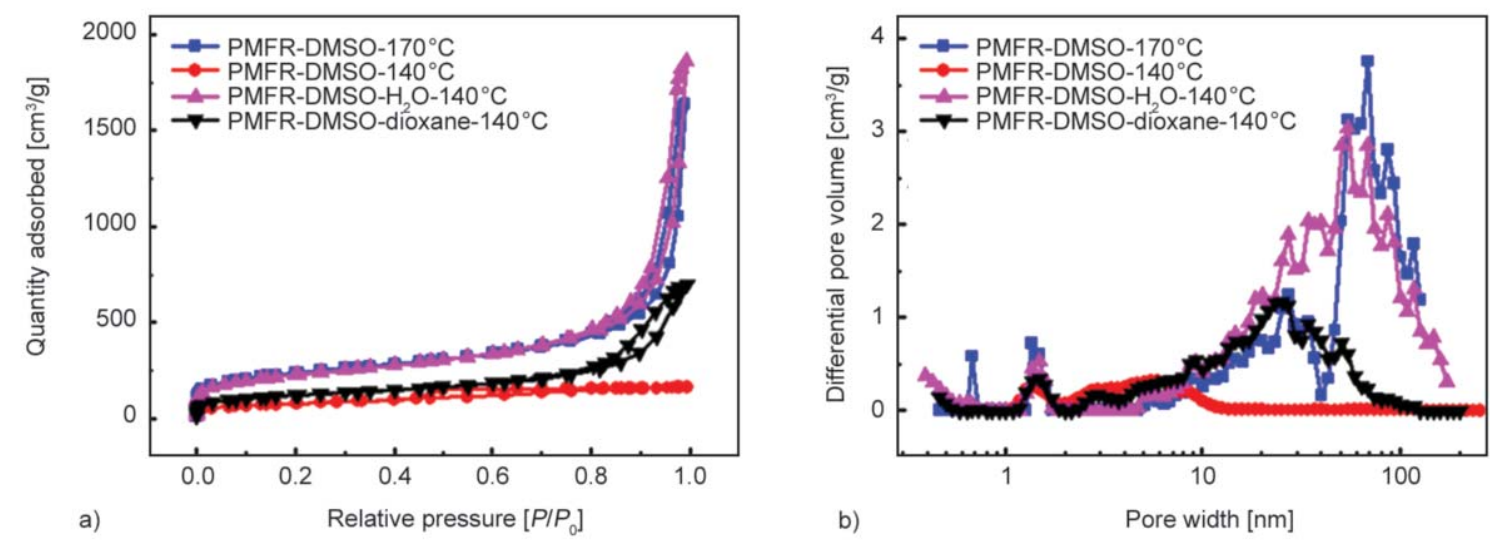

Figure 2. $\mathrm{N}_{2}$ adsorption-desorption isotherms at $77 \mathrm{~K}$ (a) and pore size distributions (b) of PMFRs

To check the pore-forming effect of the low-boilingpoint molecules, $\mathrm{H}_{2} \mathrm{O}$ or dioxane were added into the reaction system as cosolvent and the polycondesation temperature was kept at $140^{\circ} \mathrm{C}$. As can be seen in Figure 2 and Table 3, both specific surface area and porosity of PMFR-DMSO $+\mathrm{H}_{2} \mathrm{O}-140^{\circ} \mathrm{C}$ and PMFR-DMSO+Dioxane- $140^{\circ} \mathrm{C}$ were much higher than those of PMFR-DMSO- $140^{\circ} \mathrm{C}$. Especially, the specific surface area of PMFR-DMSO $+\mathrm{H}_{2} \mathrm{O}-140^{\circ} \mathrm{C}$ $\left(812 \mathrm{~m}^{2} / \mathrm{g}\right)$ is comparable to that of PMFR-DMSO$170^{\circ} \mathrm{C}\left(836 \mathrm{~m}^{2} / \mathrm{g}\right)$. This result confirms that the lowboiling-point solvents, $\mathrm{H}_{2} \mathrm{O}$ (b.p. $100^{\circ} \mathrm{C}$ ) and dioxane (b.p. $101^{\circ} \mathrm{C}$ ), can act as pore-forming agent and effectively promote the formation of pores. It is also evident that both specific surface area and pore volume of PMFR-DMSO $+\mathrm{H}_{2} \mathrm{O}-140{ }^{\circ} \mathrm{C}$ were two times as much as those of PMFR-DMSO+dioxane- $140^{\circ} \mathrm{C}$. This may be caused by the better compatibility between water and the melamine-paraformaldehyde polymers. As a result, the phase segregation delayed to the stage when the degree of crosslink was higher during the polymerization process. Such phenomenon was beneficial to forming bigger polymer particles, and thus resulted in the higher specific surface area and plentiful stacked pores. The SEM and TEM of PMFRs are shown in Figure 3 and Figure 4. SEM image of PMFR-DMSO- $170^{\circ} \mathrm{C}$ was formed by polymer particle agglomerates of various sizes. When the temperature dropped to $140^{\circ} \mathrm{C}$, the surface of PMFRDMSO- $140^{\circ} \mathrm{C}$ become smooth. However, after added the low boiling-point-solvent of water or dioxane into the system, the samples of PMFR-DMSO- $\mathrm{H}_{2} \mathrm{O}$ $140^{\circ} \mathrm{C}$ and PMFR-DMSO-dioxane- $140^{\circ} \mathrm{C}$ were made up of polymer particle again. From the TEM images, a disorder of pore structure of all PMFRs could be observed. It could be also observed that there are a lot of macropores in all samples; and plentiful of mesopores could be observed in samples employing low boilingpoint-solvent in the reaction system, especially in the sample of PMFR-DMSO- $\mathrm{H}_{2} \mathrm{O}-140{ }^{\circ} \mathrm{C}$.

According to the above observations, a scheme shown in Figure 5 is proposed to explain the poreforming mechanism of the PMFRs. In the early nucleation stage, small flakes of the polymer framework were formed by the reaction between melamine and formaldehyde (Figure 5a). With proceeding of polymerization time, the presence of low-boilingpoint solvent (such as water molecules) inside the flakes can cause the gas-liquid exchange and therefore lead to the formation of open micropores (Figure $5 b)$. In the case of high-temperature $\left(170^{\circ} \mathrm{C}\right)$, DMSO can decompose to form formaldehyde, water and dimethylsulfide, these decomposition products are expected to play the similar role as water for the formation of pores. As the further proceeding of the polymerization, the flakes grow larger and precipitate out from reactions system, causing the phase separation. The stack of these flakes can then cause the formation of mesopores and macropores (Figure 5c).

Table 3. Pore structure parameters of the PMFRs

\begin{tabular}{|l|c|c|c|}
\hline \multicolumn{1}{|c|}{ Sample } & $\begin{array}{c}\text { BET specific surface area } \\
{\left[\mathbf{m}^{\mathbf{2}} / \mathbf{g}\right]}\end{array}$ & $\begin{array}{c}\text { Pore volume } \\
{\left[\mathbf{c m}^{\mathbf{3}} / \mathbf{g}\right]}\end{array}$ & $\begin{array}{c}\text { Average pore width } \\
{[\mathbf{n m}]}\end{array}$ \\
\hline PMFR-DMSO- $170^{\circ} \mathrm{C}$ & 836 & 2.53 & 12.1 \\
\hline PMFR-DMSO- $140^{\circ} \mathrm{C}$ & 277 & 0.25 & 3.6 \\
\hline PMFR-DMSO $+\mathrm{H}_{2} \mathrm{O}-140^{\circ} \mathrm{C}$ & 812 & 2.88 & 14.2 \\
\hline PMFR-DMSO + dioxane- $140^{\circ} \mathrm{C}$ & 429 & 1.08 & 10.1 \\
\hline
\end{tabular}




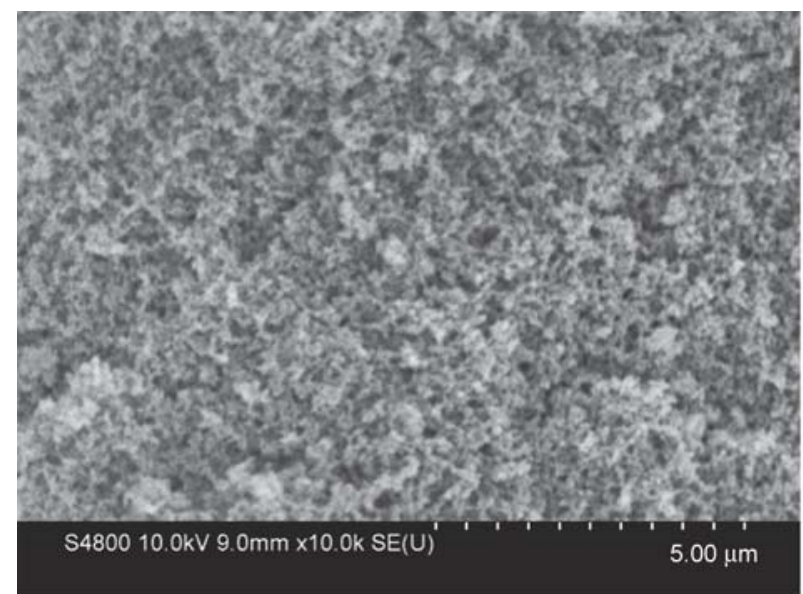

a)

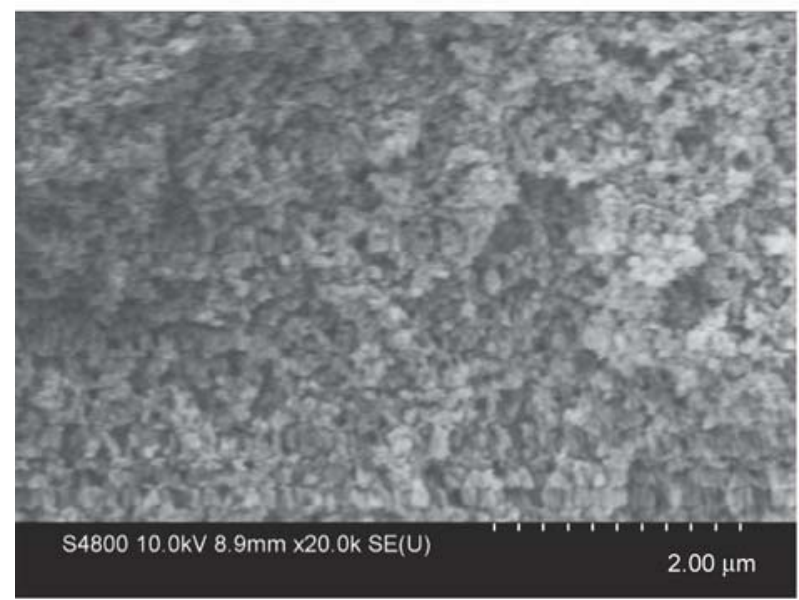

c)

PMFR-DMSO- $\mathrm{H}_{2} \mathrm{O}-140^{\circ} \mathrm{C}$

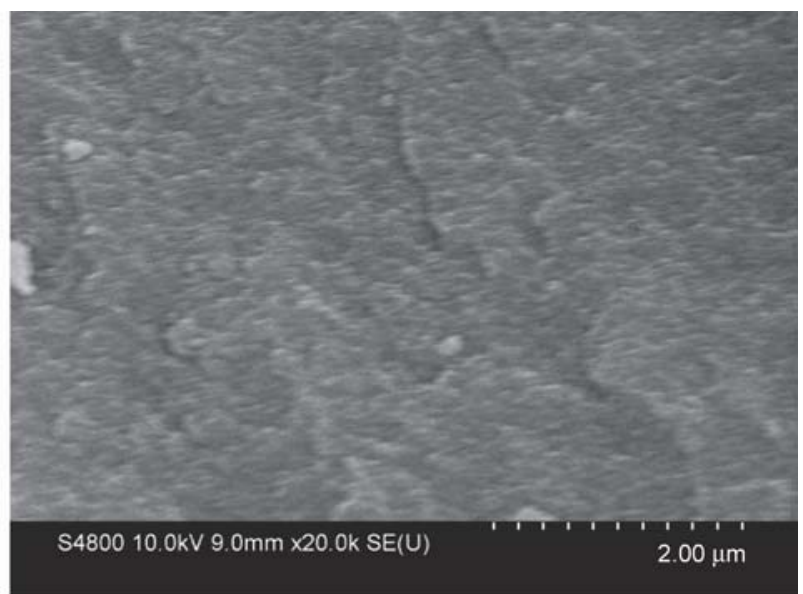

b) PMFR-DMSO- $140^{\circ} \mathrm{C}$

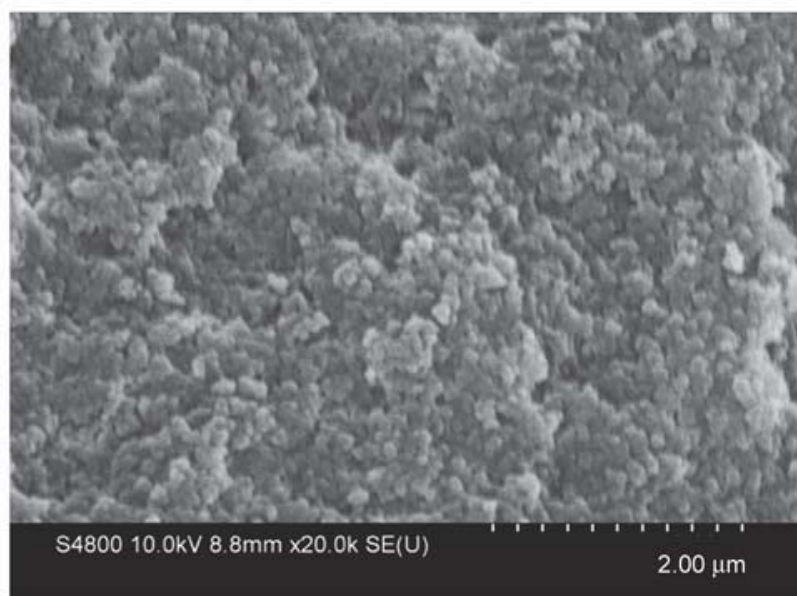

PMFR-DMSO-dioxane- $140^{\circ} \mathrm{C}$

Figure 3. SEM images of PMFRs, a) PMFR-DMSO- $170^{\circ} \mathrm{C}$, b) PMFR-DMSO- $140^{\circ} \mathrm{C}$, c) PMFR-DMSO $+\mathrm{H}_{2} \mathrm{O}-140{ }^{\circ} \mathrm{C}$, d) PMFR-DMSO+dioxane- $140^{\circ} \mathrm{C}$

\subsection{Optimizing the preparation condition of PMFRs}

Numbers of low-boiling-point solvents, such as methanol (b.p. $64.5^{\circ} \mathrm{C}$ ) and ethanol (b.p. $78^{\circ} \mathrm{C}$ ), were also used in the synthesis of PMFRs. However, the synthesized PMFRs showed low specific surface area and poor porosity (173 and $154 \mathrm{~m}^{2} / \mathrm{g}$ ). This result proved that when the boiling point of the solvent is far below the synthesis temperature, it would evaporate much more easily, and escape from the polymer, and therefore it can not act as the pore forming template.

Among these low-boiling-point solvent $\left(\mathrm{H}_{2} \mathrm{O}\right.$, dioxane, methanol and ethanol), $\mathrm{H}_{2} \mathrm{O}$ showed the most excellent role of pore-forming. Herein, we briefly optimized the preparation condition by using $\mathrm{H}_{2} \mathrm{O}$ as low-boiling-point solvent. In order to investigate the effect of water content in the mix solvent on the pore forming of PMFR, volume ratio of water to DMSO was adjusted from 0:20 to 10:10. It is found that the amount of low-boiling-point solvent could significantly affect the formation of pores. As shown in Figure 6a, with the increase of water proportion in the mixed solvents, the specific surface area and the pore volume of the obtained PMFRs increased significantly first and then decreased gradually. The decrease of specific surface area and pore volume should be ascribed to the fact that the increase of water content actually reduces the volume of a liquid solvent in the polymerization system at $140^{\circ} \mathrm{C}$, which impels the condensation polymers to separate from the solvent phase in the early stage. The small polymer particles with lower crosslinking degree would tightly stack together, thus less pores were formed. As a result, the PMFRs synthesized with over-high water proportion would have a lower specific surface area and a lower pore volume.

Except boiling point and content of low-boilingpoint solvent, the effect of the synthesis temperature, the monomer concentration and the molar ratio of paraformaldehyde to melamine on the porosities of the obtained PMFRs were carefully studied. As shown 


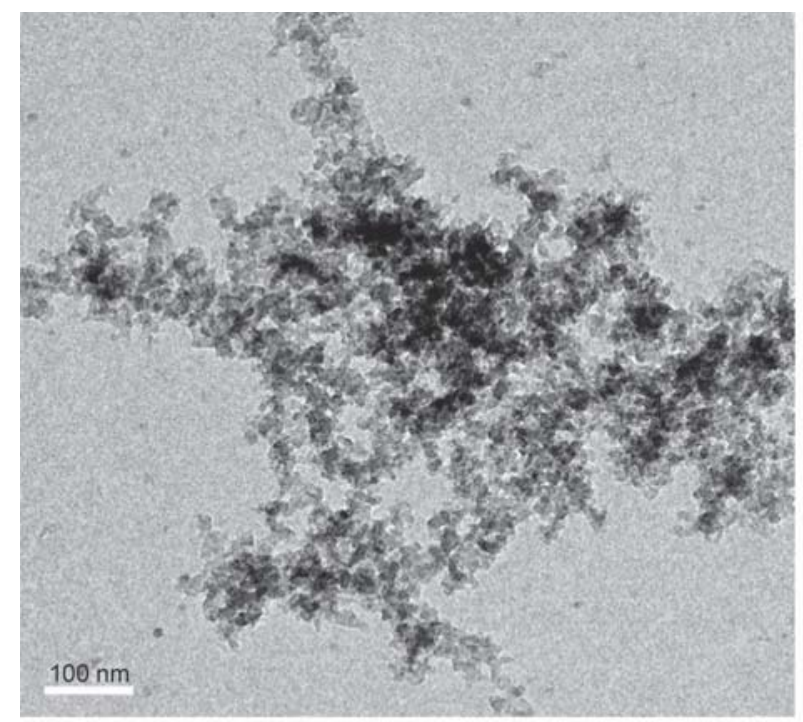

a)

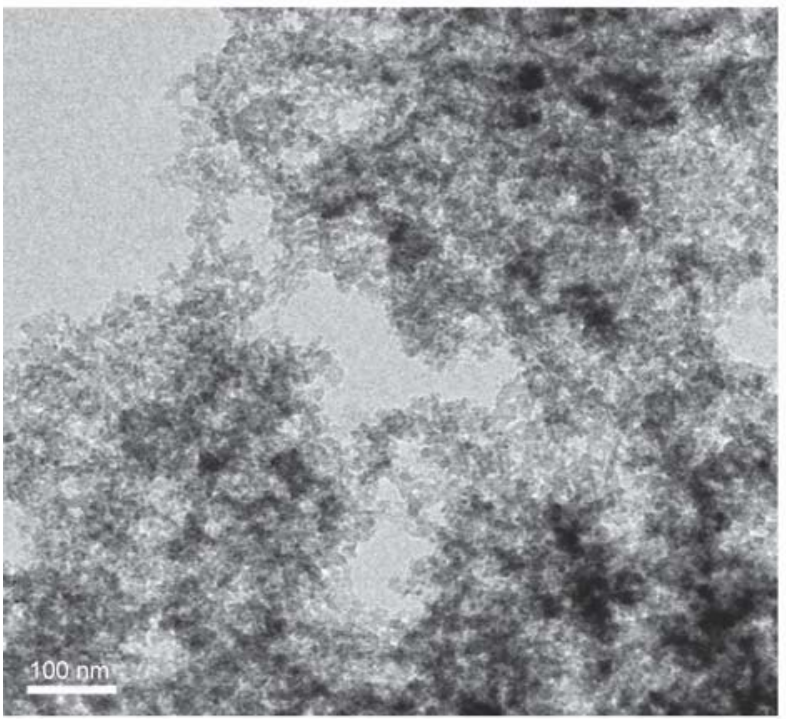

c)

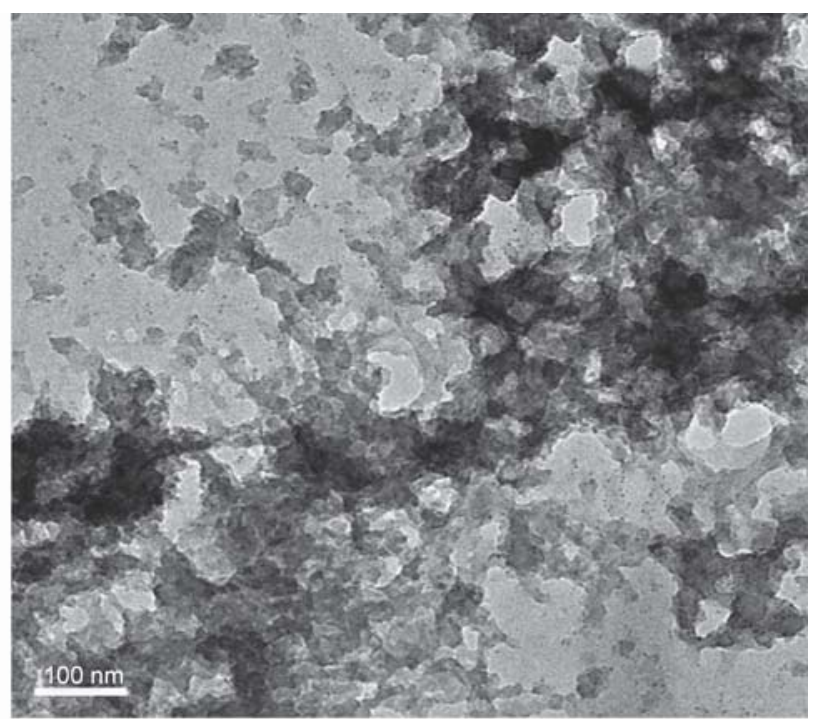

b)

PMFR-DMSO- $140^{\circ} \mathrm{C}$

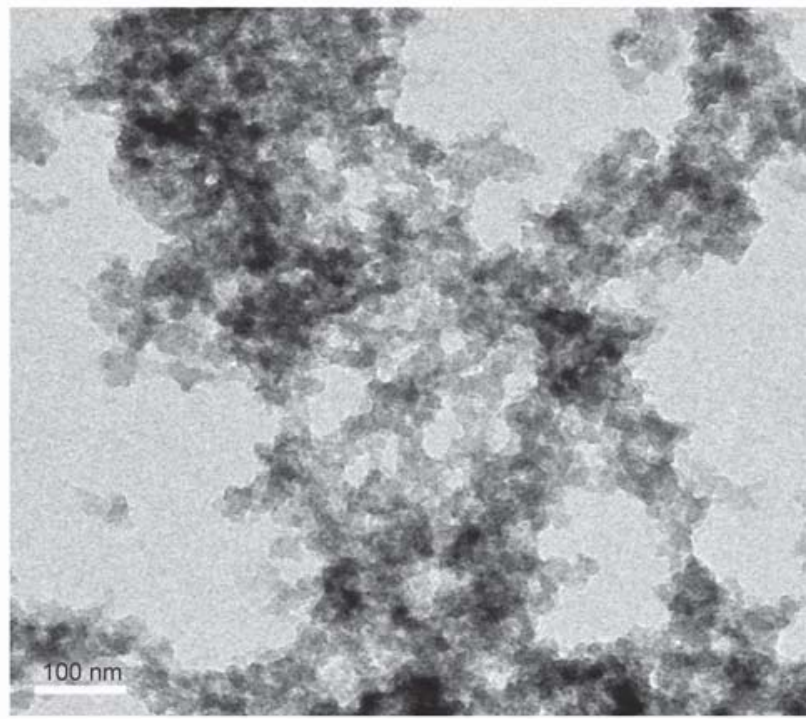

d)

PMFR-DMSO-dioxane- $140^{\circ} \mathrm{C}$

Figure 4. TEM images of PMFRs, a) PMFR-DMSO- $170^{\circ} \mathrm{C}$, b) PMFR-DMSO- $140{ }^{\circ} \mathrm{C}$, c) PMFR-DMSO $+\mathrm{H}_{2} \mathrm{O}-140^{\circ} \mathrm{C}$, d) PMFR-DMSO+dioxane- $140{ }^{\circ} \mathrm{C}$

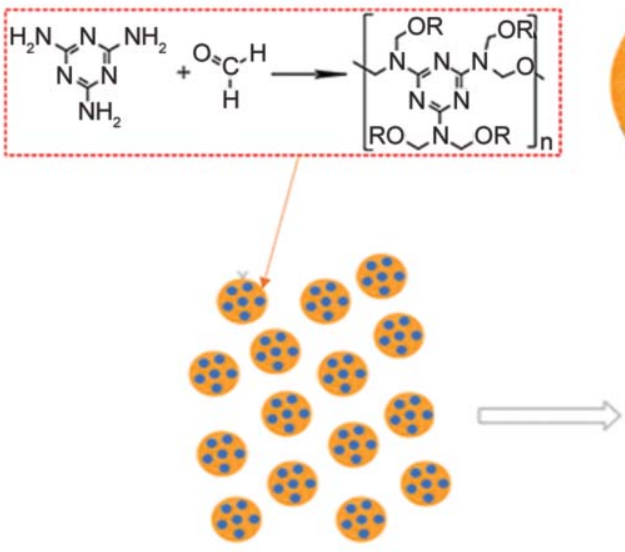

a)

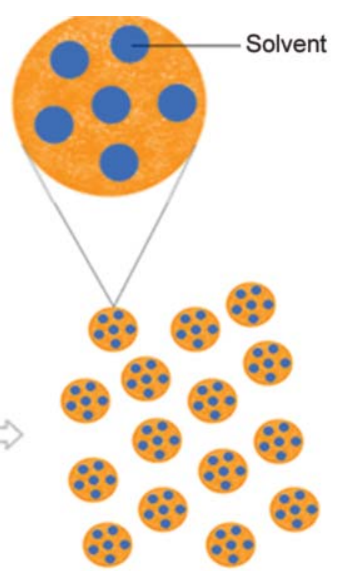

b)

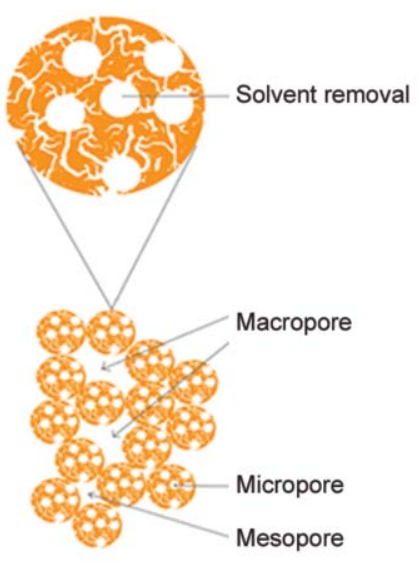

c)

Figure 5. Illustration of the effect of low-boiling point solvent in pore formation of PMFRs, (a) polycondensation of melamine and formaldehyde to form small flake, (b) solvent volatilization to form micropores, (c) stack of flakes to form mesopores and macropores 

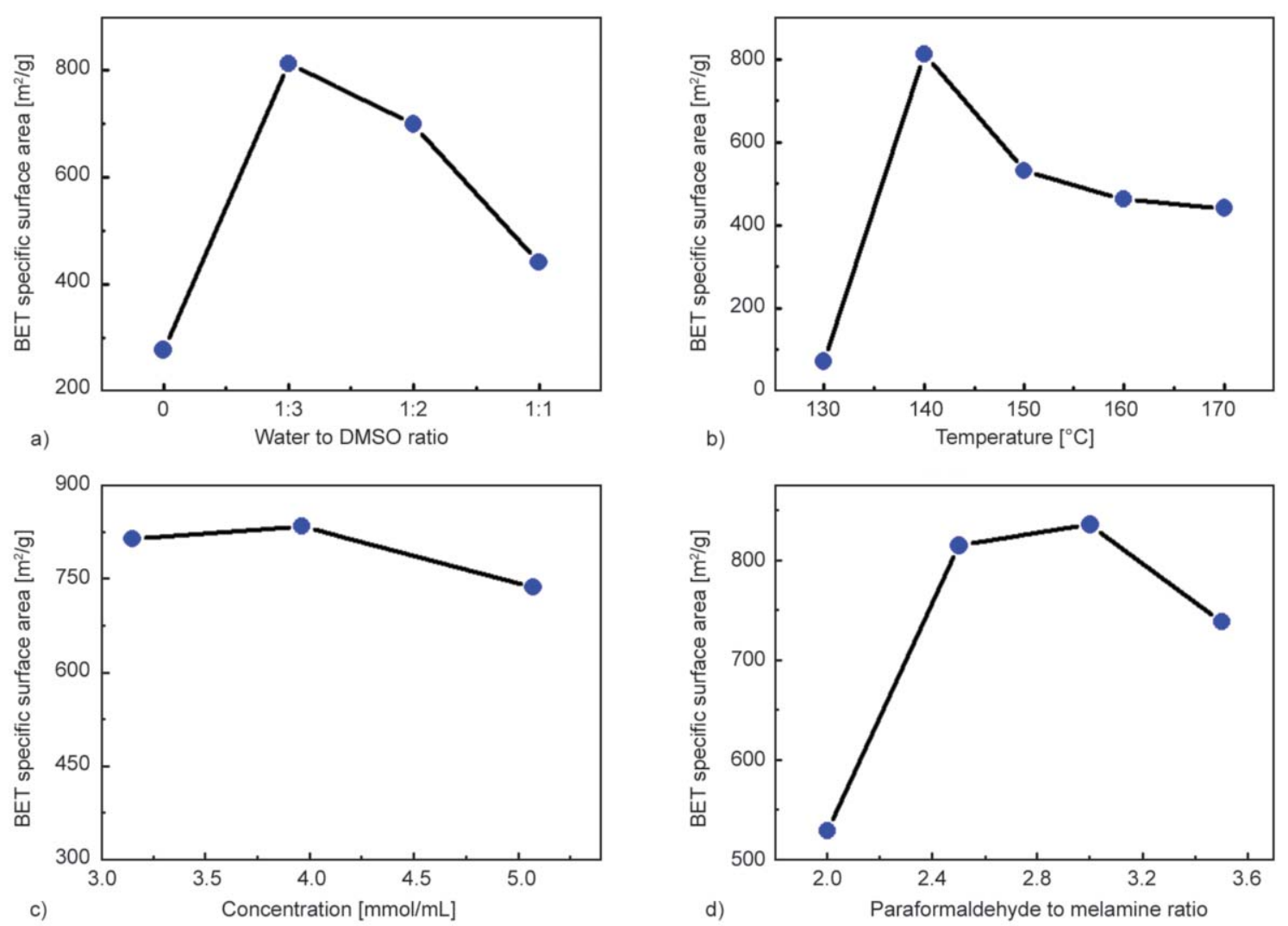

Figure 6. Effect of water to DMSO volume ratio (a), synthesis temperature (b), monomer concentration (c) and paraformaldehyde to melamine molar ratio (d) on the specific surface area of PMFRs

in Figure 6b, the PMFR prepared with a water to DMSO ratio1:3 showed the largest specific surface area when synthesized at $\sim 140^{\circ} \mathrm{C}$. Increasing or decreasing the synthesis temperature could both lead to the reduction of the specific surface area. It should be pointed out that, the PMFRs prepared in the mixed solvent of DMSO and water at around $\sim 170^{\circ} \mathrm{C}$ had much lower specific surface area than those synthesized in pure DMSO at the same temperature (PMFRDMSO $\left.-170^{\circ} \mathrm{C}\right)$. This may be due to the following: an excessively high temperature would cause violent fluid-air exchange and hence reduces the opportunity of polymer wrapping the small molecules, while a too low temperature can suppress the fluid-air exchange and hinder the small molecules to act as poreforming agent in the synthesis.

The influence of monomer concentration (total molar amount of paraformaldehyde and melamine monomers in the whole mix solution $[\mathrm{mmol} / \mathrm{mL}]$ ) on the BET specific surface area of PMFRs was studied. Figure $6 \mathrm{c}$ shows the BET specific surface area of PMFRs prepared by different monomer concentration. It can be seen from Figure $6 \mathrm{c}$ that there was no apparent change in BET specific surface area of PMFRs when the monomer concentration was kept in the range of 3.15 to $5.0 \mathrm{mmol} / \mathrm{mL}$. This result suggests that the monomer concentration does not have significant influence on the framework of PMFRs. Therefore, the monomer concentration was chosen to be $3.15 \mathrm{mmol} / \mathrm{L}$.

The effect of paraformaldehyde to melamine $(\mathrm{F} / \mathrm{M})$ molar ratio on the pore structure of PMFRs is also investigated. As shown in Figure 6d, the materials with the highest BET specific surface area and largest total pore volume were obtained with the $\mathrm{F} / \mathrm{M}$ molar ratio of 2.5. Both increasing and decreasing the $F / M$ molar ratio can lead to the decrease of the pore structure parameter. It is well known that the crosslinking site could be scarce when the concentration of paraformaldehyde is low, resulting in a low structural rigidity of amino resins and the following collapse of the pores. There the BET specific surface area and total pore volume were small when the molar ratio was smaller than 2.5. As the molar ratio increased to 3.5, polymers with decreased pore size and total pore volume were obtained. This could be attributed to the fact that the overweight addition of paraformaldehyde will result in Cannizzaro reaction and determine the $\mathrm{pH}$ of system, which will accelerate the condensation of the monomer and weaken the effect of 
the hole forming of solvent. Moreover, because of the increasing of crosslinking site, the phase separation occurred at early an stage of the polymerization when the monomer conversion rate is low. Particles with small diameter tend to form polymers with smaller pore diameter. These results suggest that the molar ratio of $\mathrm{F} / \mathrm{M}$ plays a significant role in the formation of pore structure. In order to obtain a high enough BET specific surface area, to protect the environment and reduce the cost, the molar ratio is chosen to be 2.5 .

\section{2. $\mathrm{CO}_{2}$ adsorption performance of PEI supported on PMFRs}

The above synthesized porous amine resin was used as support, and PEI was loaded to prepared a solid amine adsorbent (PMFR-PEI) for $\mathrm{CO}_{2}$ capture. The $\mathrm{CO}_{2}$ adsorption properties of PMFR and PMFR impregnated with different amounts of PEI were evaluated by measuring the $\mathrm{CO}_{2}$ breakthrough curves. As shown in Figure 7, the breakthrough curve could be divided into three phases: phase 1, complete adsorption phase at the beginning, $\mathrm{CO}_{2}$ can be completely adsorbed in this phase due to the large number of active sites; phase 2, breakthrough phase, $\mathrm{CO}_{2}$ partially begun to breakthrough the fixed bed; and (3) phase 3 , saturation phase, $\mathrm{CO}_{2}$ has fully occupied of the active sites and no more $\mathrm{CO}_{2}$ can be adsorbed [38-40]. Comparison of the three adsorption phases of PMFR-PEIs with those of the solid amine fibers indicated that the breakthrough time of PMFR-PEI at phase 2 was much faster than solid amine fiber, it took only 2 to 3 minutes from the initial breakthrough phase to the saturation phase. This result indicates that PMFR-PEIs has a much superior mass transfer and adsorption kinetics due to the presence of pores. Different breakthrough behaviors of PMFR and

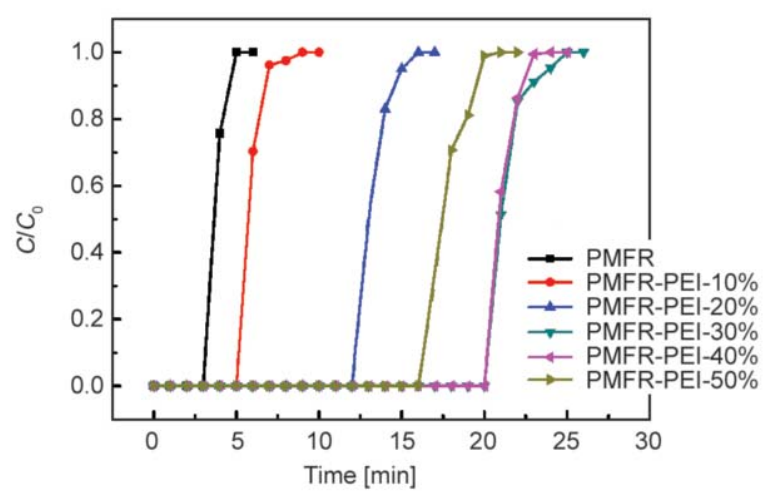

Figure 7. Effect of PEI loading on the $\mathrm{CO}_{2}$ adsorption capacity
PMFR-PEI samples with different PEI loading amount were also observed. The $\mathrm{CO}_{2}$ adsorption capacity of PMFR was only $0.47 \mathrm{mmol} / \mathrm{g}$, while the $\mathrm{CO}_{2}$ adsorption capacity of PMFR-PEI increased from 0.86 to $2.89 \mathrm{mmol} / \mathrm{g}$ when the amount of PEI loading increased from 10 to $30 \%$ (Table 4 ) and the highest $\mathrm{CO}_{2}$ adsorption capacity of PMFR-PEI was much higher than that of PMFR, indicating that the loading PEI can effectively improve the adsorption capacity and more PEI loading amount can provide more amino site for $\mathrm{CO}_{2}$ adsorption. However, the $\mathrm{CO}_{2}$ adsorption capacity decreased when the PEI loading amount was further increased to $50 \%$, which could be due to the serious pore blocking and to the increase of the mass transfer resistance.

The amino content could be calculated by diving the loading weight of PEI with molecular weight of the chain segments of PEI, as shown in Equation (2):

Amino content $[\mathrm{mmol} / \mathrm{g}]=\frac{W_{\mathrm{PEE}} \cdot 1000}{42}$

where $W_{\mathrm{PEI}}$ is PEI loading amount (g PEI/g adsorbent) in the adsorbent, 42 is the molecular weight of the chain segments of PEI. Therefore, the utilization efficiency of amino could be evaluated by dividing the actual $\mathrm{CO}_{2}$ adsorption capacity $[\mathrm{mmol} / \mathrm{g}$ ] with amino content $[\mathrm{mmol} / \mathrm{g}]$ of the adsorbent, supposing that one mole amino can combine with one mole $\mathrm{CO}_{2}$. For the branched PEI, percentages of primary, secondary and tertiary amino groups are about 35 , 35 , and $30 \%$, respectively. It has been reported that at the absence of water, one acidic $\mathrm{CO}_{2}$ molecule will combine with two primary or secondary amino groups within the PEI molecules. $\mathrm{CO}_{2}$ molecule can not combine with tertiary amino group in the absence of water [41]. Thus the theoretical maximum amino utilization efficiency of the branched PEI is about 35\% [42-44]. The results in this study showed

Table 4. $\mathrm{CO}_{2}$ adsorption properties of PMFR-PEI with different PEI loading

\begin{tabular}{|c|c|c|c|c|}
\hline $\begin{array}{c}\text { PEI loading } \\
\text { amount } \\
{[\mathbf{w t} \%]}\end{array}$ & $\begin{array}{c}\boldsymbol{S}_{\mathrm{BET}} \\
{\left[\mathbf{m}^{\mathbf{2}} \mathbf{g} \mathbf{g}\right]}\end{array}$ & $\begin{array}{c}\text { Adsorption } \\
\text { capacity } \\
{[\mathbf{m m o l} / \mathbf{g}]}\end{array}$ & $\begin{array}{c}\text { Amino } \\
\text { content } \\
{[\mathbf{m m o l} / \mathbf{g}]}\end{array}$ & $\begin{array}{c}\text { Amino } \\
\text { utilization } \\
\text { efficiency } \\
{[\%]}\end{array}$ \\
\hline 0 & 812 & 0.47 & 0.00 & 0.0 \\
\hline 10 & 424 & 0.86 & 2.38 & 36.1 \\
\hline 20 & 309 & 1.76 & 4.76 & 37.0 \\
\hline 30 & 212 & 2.89 & 7.14 & 40.5 \\
\hline 40 & 130 & 2.82 & 9.52 & 29.6 \\
\hline 50 & 50 & 2.37 & 11.90 & 19.9 \\
\hline
\end{tabular}



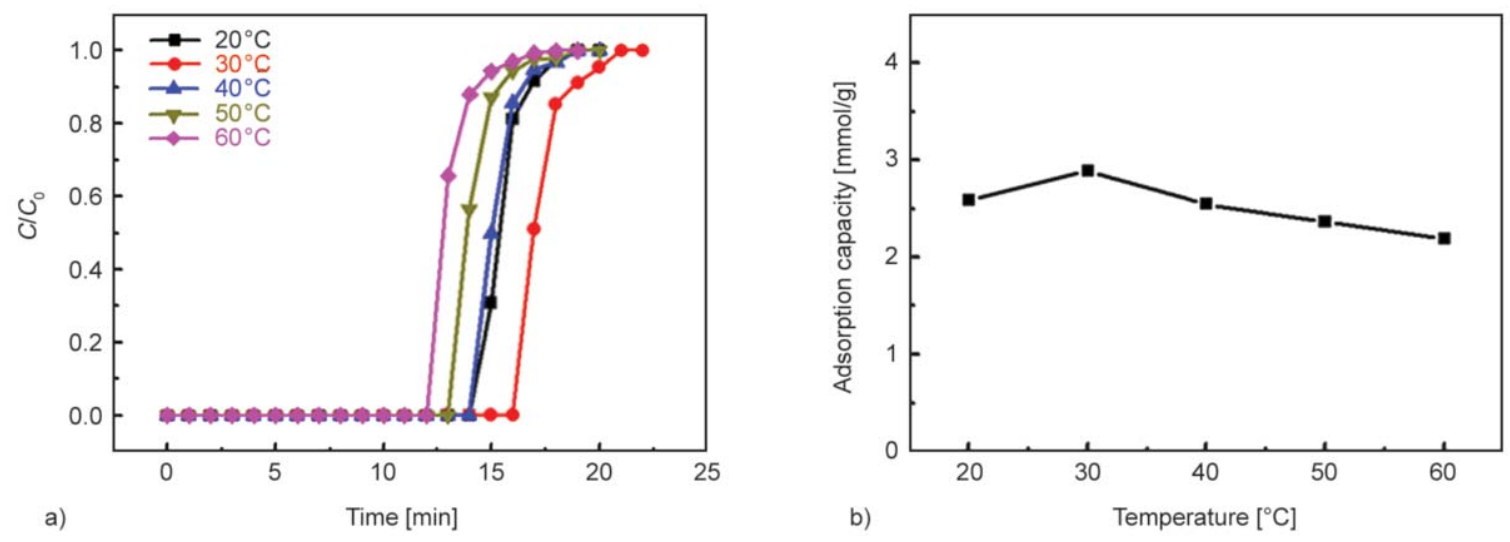

Figure 8. Breakthrough curves (a) and capacity (b) of $\mathrm{CO}_{2}$ adsorption on PMFR-PEI-30\% at different temperatures

that the amino utilization efficiency of PMFR-PEI was quite close to or exceeded its theoretical value when the PEI loading amount was less than 30\% (Table 4). For example, the amino utilization efficiency was $40.5 \%$ when the PEI loading amount was $30 \%$, which should be ascribed to the synergistic effect between the physisorption of pore and the chemisorption of loading PEI. However, when further increasing the PEI to $50 \%$, the amino utilization efficiency evidently decreased. This fact suggested that the full loading of PEI into the pores of PMFR would increase the layer thickness of PEI, thus may inhibit mass transfer. As a result, part of PEI may not be able to contact and adsorb $\mathrm{CO}_{2}$, resulting in the decrease of amino utilization efficiency.

The influence of temperature on the $\mathrm{CO}_{2}$ adsorption of PMFR-PEI-30\% (30\% PEI loading) was investigated (Figure 8). It is obvious that the PMFR-PEI$30 \%$ adsorbent showed a maximum adsorption capacity of $2.89 \mathrm{mmol} / \mathrm{g}$ at $30^{\circ} \mathrm{C}$, implying the balance between $\mathrm{CO}_{2}$ diffusion into inner layer of PEI and the desorption of $\mathrm{CO}_{2}$ from PEI $[43,45]$. The adsorption process at a lower temperature was diffusion controlled, elevation of temperature would accelerate the diffusion of $\mathrm{CO}_{2}$ inside of the thick layer of the PEI, and be beneficial for the full extension of the PEI's chain and hence provide more adsorption sites, leading to more sufficient contact with $\mathrm{CO}_{2}$. On the other hand, elevating temperature would also accelerate the decomposition of the ammonium carbonate, which is unfavorable to the adsorption. It was noted that PMFR-PEI-30\% can still have a $\mathrm{CO}_{2}$ adsorption capacity of $2.19 \mathrm{mmol} / \mathrm{g}$ even at $60^{\circ} \mathrm{C}$, there was only a small decrement of $0.7 \mathrm{mmol} / \mathrm{g}$ compared with that at $30^{\circ} \mathrm{C}$.

For the purpose of evaluating the regeneration performance of the PMFR-PEI-30\%, the PMFR-PEI-

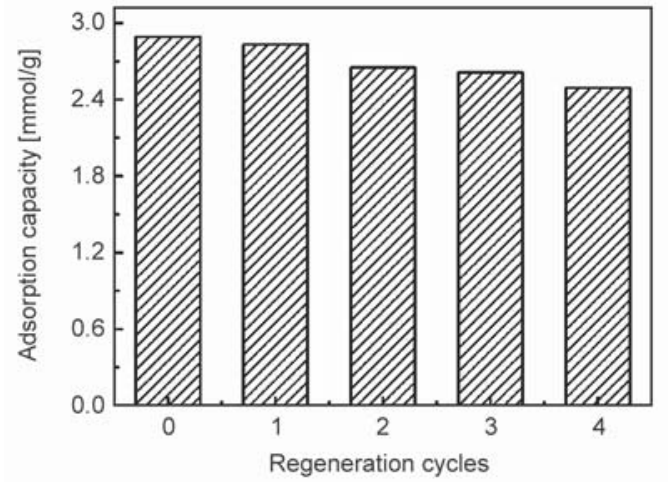

Figure 9. Adsorption capacities of $\mathrm{CO}_{2}$ on fresh and regenerated PMFR-PEI-30\%

$30 \%$ was regenerated by heating it to $90{ }^{\circ} \mathrm{C}$ in a $30 \mathrm{~mL} / \mathrm{min}$ dry $\mathrm{N}_{2}$ stream, and the results are shown in Figure 9. The regenerated PMFR-PEI-30\% can keep over $85 \%$ capacity of the fresh PMFR-PEI-30\% adsorbent after four adsorption-desorption cycles.

\section{Conclusions}

A simple approach of using low-boiling-point solvents as pore-forming reagents was developed to synthesize porous melamine-formaldehyde resin under much less rigorous conditions $\left(140^{\circ} \mathrm{C}\right.$ and $20 \mathrm{~h}$ ). A comparable high specific surface area as those synthesized under tough conditions $\left(170^{\circ} \mathrm{C}, 72 \mathrm{~h}\right)$ could be achieved by using this approach. It was found that the presence of low-boiling-point solvent (such as water) wrapped inside the polymer particles could lead to the formation of micropores, thus increased the porosity of the prepared melamine-formaldehyde resin. When loading with 30\% PEI, the PMFRs synthesized by this method under optimal conditions demonstrated good $\mathrm{CO}_{2}$ adsorption capacity up to $2.89 \mathrm{mmol} / \mathrm{g}$ at $30^{\circ} \mathrm{C}$. These results are helpful for understanding the pore-forming process of PMFRs, which are useful for the development of 
new and environmentally-friendly synthetic methods for high specific surface area porous materials.

\section{Acknowledgements}

The authors gratefully acknowledge the financial support provided by the National Natural Science Foundation of China (Grant No. 51473187), Natural Science Foundation of Guangdong Province (2016A010103013).

\section{References}

[1] Gomes R., Bhanja P., Bhaumik A.: A triazine-based covalent organic polymer for efficient $\mathrm{CO}_{2}$ adsorption. Chemical Communications, 51, 10050-10053 (2015). https://doi.org/10.1039/C5CC02147B

[2] Varghese O. K., Paulose M., LaTempa T. J., Grimes C. A.: High-rate solar photocatalytic conversion of $\mathrm{CO}_{2}$ and water vapor to hydrocarbon fuels. Nano Letters, 9 , 731-737 (2009). https://doi.org/10.1021/nl803258p

[3] Tu W., Zhou Y., Zou Z.: Photocatalytic conversion of $\mathrm{CO}_{2}$ into renewable hydrocarbon fuels: State-of-the-art accomplishment, challenges, and prospects. Advanced Materials, 26, 4607-4626 (2014). https://doi.org/10.1002/adma.201400087

[4] Nugent P., Belmabkhout Y., Burd S. D., Cairns A. J., Luebke R., Forrest K., Pham T., Ma S., Space B., Wojtas L., Eddaoudi M., Zaworotko M. J.: Porous materials with optimal adsorption thermodynamics and kinetics for $\mathrm{CO}_{2}$ separation. Nature, 495, 80-84 (2013). https://doi.org/10.1038/nature11893

[5] Salles F., Jobic H., Devic T., Llewellyn P. L., Serre C., Férey G., Maurin G.: Self and transport diffusivity of $\mathrm{CO}_{2}$ in the metal-organic framework MIL-47(V) explored by quasi-elastic neutron scattering experiments and molecular dynamics simulations. ACS Nano, 4, 143-152 (2010). https://doi.org/10.1021/nn901132k

[6] Kong L., Zou R., Bi W., Zhong R., Mu W., Liu J., Han R. P. S., Zou R.: Selective adsorption of $\mathrm{CO}_{2} / \mathrm{CH}_{4}$ and $\mathrm{CO}_{2} / \mathrm{N}_{2}$ within a charged metal-organic framework. Journal of Materials Chemistry A, 2, 17771-17778 (2014). https://doi.org/10.1039/C4TA01993H

[7] Vlaisavljevich B., Odoh S. O., Schnell S. K., Dzubak A. L., Lee K., Planas N., Neaton J. B., Gagliardi L., Smit B.: $\mathrm{CO}_{2}$ induced phase transitions in diamine-appended metal-organic frameworks. Chemical Science, 6, 5177 5185 (2015). https://doi.org/10.1039/C5SC01828E

[8] Sumida K., Rogow D. L., Mason J. A., McDonald T. M., Bloch E. D., Herm Z. R., Bae T-H., Long J. R.: Carbon dioxide capture in metal-organic frameworks. Chemical Reviews, 112, 724-781 (2012).

https://doi.org/10.1021/cr2003272
[9] Montazerolghaem M., Aghamiri S. F., Tangestaninejad S., Talaie M. R.: A metal-organic framework MIL-101 doped with metal nanoparticles ( $\mathrm{Ni}$ and $\mathrm{Cu}$ ) and its effect on $\mathrm{CO}_{2}$ adsorption properties. RSC Advances, 6, 632-640 (2016).

https://doi.org/10.1039/C5RA22450k

[10] Janiak C., Henninger S. K.: Porous coordination polymers as novel sorption materials for heat transformation processes. Chimia, 67, 419-424 (2013). https://doi.org/10.2533/chimia.2013.419

[11] Li P., He Y., Guang J., Weng L., Zhao J. C-G., Xiang S., Chen B.: A homochiral microporous hydrogen-bonded organic framework for highly enantioselective separation of secondary alcohols. Journal of the American Chemical Society, 136, 547-549 (2014).

https://doi.org/10.1021/ja4129795

[12] Adeniran B., Mokaya R.: Is N-doping in porous carbons beneficial for $\mathrm{CO}_{2}$ storage? Experimental demonstration of the relative effects of pore size and N-doping. Chemistry of Materials, 28, 994-1001 (2016). https://doi.org/10.1021/acs.chemmater.5b05020

[13] Coromina H.M., Walsh D. A., Mokaya R.: Biomass-derived activated carbon with simultaneously enhanced $\mathrm{CO}_{2}$ uptake for both pre and post combustion capture applications. Journal of Materials Chemistry A, 4, 280 289 (2016).

https://doi.org/10.1039/C5TA09202G

[14] Hosseini S., Marahel E., Bayesti I., Abbasi A., Abdullah C. L., Choong T. S. Y.: $\mathrm{CO}_{2}$ adsorption on modified carbon coated monolith: Effect of surface modification by using alkaline solutions. Applied Surface Science, 324, 569-575 (2015). https://doi.org/10.1016/j.apsusc.2014.10.054

[15] Choma J., Jedynak K., Fahrenholz W., Ludwinowicz J., Jaroniec M.: Microporosity development in phenolic resin-based mesoporous carbons for enhancing $\mathrm{CO}_{2}$ adsorption at ambient conditions. Applied Surface Science, 289, 592-600 (2014).

https://doi.org/10.1016/j.apsusc.2013.11.051

[16] Bai B. C., Kim E. A., Lee C. W., Lee Y-S., Im J. S.: Effects of surface chemical properties of activated carbon fibers modified by liquid oxidation for $\mathrm{CO}_{2}$ adsorption. Applied Surface Science, 353, 158-164 (2015). https://doi.org/10.1016/j.apsusc.2015.06.046

[17] Lan J., Cao D., Wang W., Smit B.: Doping of alkali, alkaline-earth, and transition metals in covalent-organic frameworks for enhancing $\mathrm{CO}_{2}$ capture by first-principles calculations and molecular simulations. ACS Nano, 4, 4225-4237 (2010). https://doi.org/10.1021/nn100962r

[18] Zhu Y., Wan S., Jin Y., Zhang W.: Desymmetrized vertex design for the synthesis of covalent organic frameworks with periodically heterogeneous pore structures. Journal of the American Chemical Society, 137, 1377213775 (2015). https://doi.org/10.1021/jacs.5b09487 
[19] Furukawa H., Yaghi O. M.: Storage of hydrogen, methane, and carbon dioxide in highly porous covalent organic frameworks for clean energy applications. Journal of the American Chemical Society, 131, 8875-8883 (2009). https://doi.org/10.1021/ja9015765

[20] Alahakoon S. B., Thompson C. M., Nguyen A. X., Occhialini G., McCandless G. T., Smaldone R. A.: An azine-linked hexaphenylbenzene based covalent organic framework. Chemical Communications, 52, 28432845 (2016). https://doi.org/10.1039/C5CC10408D

[21] Sui Z-Y., Cui Y., Zhu J-H., Han B-H.: Preparation of three-dimensional graphene oxide-polyethylenimine porous materials as dye and gas adsorbents. ACS Applied Materials and Interfaces, 5, 9172-9179 (2013). https://doi.org/10.1021/am402661t

[22] Ko Y. G., Lee H. J., Kim J. Y., Choi U. S.: Hierarchically porous aminosilica monolith as a $\mathrm{CO}_{2}$ adsorbent. ACS Applied Materials and Interfaces, 6, 12988-12996 (2014). https://doi.org/10.1021/am5029022

[23] Schwab M. G., Fassbender B., Spiess H. W., Thomas A., Feng X., Müllen K.: Catalyst-free preparation of melamine-based microporous polymer networks through schiff base chemistry. Journal of the American Chemical Society, 131, 7216-7217 (2009).

https://doi.org/10.1021/ja902116f

[24] McDonald T. M., Lee W. R., Mason J. A., Wiers B. M., Hong C. S., Long J. R.: Capture of carbon dioxide from air and flue gas in the alkylamine-appended metal-organic framework mmen- $\mathrm{Mg}_{2}$ (dobpdc). Journal of the American Chemical Society, 134, 7056-7065 (2012). https://doi.org/10.1021/ja300034j

[25] Li G., Zhang B., Wang Z.: Facile synthesis of fluorinated microporous polyaminals for adsorption of carbon dioxide and selectivities over nitrogen and methane. Macromolecules, 49, 2575-2581 (2016).

https://doi.org/10.1021/acs.macromol.6b00147

[26] Goeppert A., Czaun M., May R. B., Prakash G. K. S., Olah G. A., Narayanan S. R.: Carbon dioxide capture from the air using a polyamine based regenerable solid adsorbent. Journal of the American Chemical Society, 133, 20164-20167 (2011). https://doi.org/10.1021/ja2100005

[27] Sayari A., Belmabkhout Y.: Stabilization of amine-containing $\mathrm{CO}_{2}$ adsorbents: Dramatic effect of water vapor. Journal of the American Chemical Society, 132, 63126314 (2010).

https://doi.org/10.1021/ja1013773

[28] Lee J. H., Lee H. J., Lim S. Y., Kim B. G., Choi J. W.: Combined $\mathrm{CO}_{2}$-philicity and ordered mesoporosity for highly selective $\mathrm{CO}_{2}$ capture at high temperatures. Journal of the American Chemical Society, 137, 7210-7216 (2015).

https://doi.org/10.1021/jacs.5b03579
[29] Yin H., Liu H., Wang W., Feng Y.: $\mathrm{CO}_{2}$-induced reversible dispersion of graphene by a melamine derivative. Langmuir, 31, 12260-12267 (2015).

https://doi.org/10.1021/acs.langmuir.5b02831

[30] Liao Y., Weber J., Faul C. F. J.: Fluorescent microporous polyimides based on perylene and triazine for highly $\mathrm{CO}_{2}$-selective carbon materials. Macromolecules, 48, 2064-2073 (2015).

https://doi.org/10.1021/ma501662r

[31] Kailasam K., Jun Y-S., Katekomol P., Epping J. D., Hong W. H., Thomas A.: Mesoporous melamine resins by soft templating of block-co-polymer mesophases. Chemistry of Materials, 22, 428-434 (2010).

https://doi.org/10.1021/cm9029903

[32] Schwarz D., Weber J.: Waterborne colloidal polymer/ silica hybrid dispersions and their assembly into mesoporous poly(melamine-formaldehyde) xerogels. Langmuir, 31, 8436-8445 (2015).

https://doi.org/10.1021/acs.langmuir.5b00990

[33] Sun G., Ma L., Ran J., Li B., Shen X., Tong H.: Templated synthesis and activation of highly nitrogen-doped worm-like carbon composites based on melamine-ureaformaldehyde resins for high performance supercapacitors. Electrochimica Acta, 194, 168-178 (2016).

https://doi.org/10.1016/j.electacta.2016.02.066

[34] Wilke A., Weber J.: Hierarchical nanoporous melamine resin sponges with tunable porosity-porosity analysis and $\mathrm{CO}_{2}$ sorption properties. Journal of Materials Chemistry, 21, 5226-5229 (2011).

https://doi.org/10.1039/C1JM10171D

[35] Tan M. X., Zhang Y., Ying J. Y.: Mesoporous poly (melamine-formaldehyde) solid sorbent for carbon dioxide capture. ChemSusChem, 6, 1186-1190 (2013). https://doi.org/10.1002/cssc.201300107

[36] Mesangeau C., Yous S., Peres B., Lesieur D., Besson T.: Pictet-spengler heterocyclizations via microwave-assisted degradation of DMSO. ChemInform, 36, 24652468 (2005). https://doi.org/10.1002/chin.200531140

[37] Yang G., Han H., Du C., Luo Z., Wang Y.: Facile synthesis of melamine-based porous polymer networks and their application for removal of aqueous mercury ions. Polymer, 51, 6193-6202 (2010). https://doi.org/10.1016/j.polymer.2010.10.052

[38] Bollini P., Didas S. A., Jones C. W.: Amine-oxide hybrid materials for acid gas separations. Journal of Materials Chemistry, 21, 15100-15120 (2011). https://doi.org/10.1039/C1JM12522B

[39] Samanta A., Zhao A., Shimizu G. K. H., Sarkar P., Gupta R.: Post-combustion $\mathrm{CO}_{2}$ capture using solid sorbents: A review. Industrial and Engineering Chemistry Research, 51, 1438-1463 (2012).

https://doi.org/10.1021/ie200686q

[40] Choi S., Drese J. H., Jones C. W.: Adsorbent materials for carbon dioxide capture from large anthropogenic point sources. ChemSusChem, 2, 796-854 (2009). https://doi.org/10.1002/cssc.200900036 
[41] Ko Y. G., Shin S. S., Choi U. S.: Primary, secondary, and tertiary amines for $\mathrm{CO}_{2}$ capture: Designing for mesoporous $\mathrm{CO}_{2}$ adsorbents. Journal of Colloid and Interface Science, 361, 594-602 (2011).

https://doi.org/10.1016/j.jcis.2011.03.045

[42] Yang Z-Z., He L-N., Gao J., Liu A-H., Yu B.: Carbon dioxide utilization with $\mathrm{C}-\mathrm{N}$ bond formation: Carbon dioxide capture and subsequent conversion. Energy and Environmental Science, 5, 6602-6639 (2012).

https://doi.org/10.1039/C2EE02774G

[43] Pinto M. L., Mafra L., Guil J. M., Pires J., Rocha J.: Adsorption and activation of $\mathrm{CO}_{2}$ by amine-modified nanoporous materials studied by solid-state NMR and ${ }^{13} \mathrm{CO}_{2}$ adsorption. Chemistry of Materials, 23, 1387 1395 (2011).

https://doi.org/10.1021/cm1029563
[44] Dillon E. P., Crouse C. A., Barron A. R.: Synthesis, characterization, and carbon dioxide adsorption of covalently attached polyethyleneimine-functionalized single-wall carbon nanotubes. ACS Nano, 2, 156-164 (2008).

https://doi.org/10.1021/nn7002713

[45] Monazam E. R., Spenik J., Shadle L. J.: Fluid bed adsorption of carbon dioxide on immobilized polyethylenimine (PEI): Kinetic analysis and breakthrough behavior. Chemical Engineering Journal, 223, 795-805 (2013). https://doi.org/10.1016/j.cej.2013.02.041 